EPJ Web of Conferences 23, 00002 (2012)

DOI: $10.1051 /$ epjconf/20122300002

(C) Owned by the authors, published by EDP Sciences, 2012

\title{
Explanation of a strange bulk-edge equality
}

\author{
R. Shankar ${ }^{1, a}$ Ashvin Vishwanath ${ }^{2}$ \\ 1 Yale University, New Haven CT 06520 \\ 2 University of California, Berkeley CA 94720
}

\begin{abstract}
In the infrared limit the $\mathrm{N}$-particle ground-state wavefunctions of the bulk happen to be exactly equal to the $\mathrm{N}$-point space-time correlation functions for certain topological superconductors. We explain this [1], beginning with the $\mathrm{p}+\mathrm{ip}$ state in $\mathrm{D}=2+1$. We write $Z(J)$, the generating function for wavefunctions, as a path Euclidean integral. Varying the chemical potential as a function of Euclidean time between weak and strong pairing states is shown to extract the wavefunction. Upon a Euclidean rotation that exchanges time and space, approximate Lorentz invariance converts the system to one with a spatial edge and $Z(J)$ to the generator of spacetime correlation functions for the edge fields. We also provide a $\mathrm{D}=3+1$ example, superfluid ${ }^{3} \mathrm{He}-\mathrm{B}$, and a pwave superfluid in $\mathrm{D}=1+1$. Our method works only when particle number is not conserved, as in superconductors.
\end{abstract}

\section{Life at the edge}

The boundaries or edges of condensed matter systems received scant attention until recent developments showed them to be fertile areas of research both in the Fractional Quantum Hall Effect (FQHE) [1-3]. and in topological insulators and superconductors $[4,5,7,6,8-10]$. These works established that in certain topological insulators (which includes superconductors because they are gapped in the bulk) the edges or boundaries carry gapless excitations which are unusual. The edge modes are saved from acquiring a gap due to various impurities because of the underlying topological quantum number. The edge modes in a $d-1$ dimensional boundary of a bulk system in $d$ dimensions cannot exist in generic $d-1$ dimensional systems that were not boundaries. For example the edge state at the $d=2$ surface of a certain $d=3$ insulator is described at low energies by a single Dirac cone, while a generic $d=2$ system

a e-mail: r.shankar@yale.edu

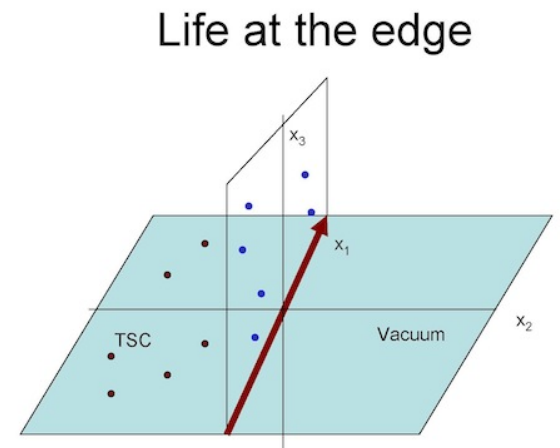

The edge is gapless, chiral or helical, fractionalized, topologically protected

Fig. 1. The $d=2$ bulk with a $d=1$ edge in the horizontal plane. The vertical plane perpendicular to the edge is its Euclidean spacetime. The dots in both planes denote the arguments of the wavefunction or correlation function. like graphene must have an even number (which happens to be 2). Fig. 1 shows a $d=2$ system with a $d=1$ edge and the edge's $1+1$ dimensional euclidean spacetime.

In two spatial dimensions, the edge dynamics is described by conformal field theory [3] which was also used to produce wave functions in the bulk $[12,11]$. Moore and Read[11] showed that one may view the FQHE wavefunctions and the quasi-hole excitations as conformal blocks in which both electrons and the quasiparticle coordinates are treated on the same footing and their charges and braiding properties are severely constrained. For an exhaustive review of many related topics see Nayak et al [13].

We discuss problems where the N-particle ground-state wavefunctions of the bulk happen to be exactly equal to the $\mathrm{N}$-point space-time correlation functions at the edge, in the infrared limit. What are the minimal ingredients necessary to establish this equality? Are analytic functions or $\mathrm{d}=2$ conformal invariance required? We show that our edgebulk equality follows for a class of topological superconductors in various dimensions invoking only approximate Lorentz symmetry. The connections obtained here using an effective low energy hamiltonian differ from CS theory[14] in which the hamiltonian vanishes and only non-dynamical particles enter via Wilson loops, as reviewed in Ref.[13].

To relate wavefunctions, which are defined at equal time, to spacetime edge correlations, it is convenient to use the Euclidean path integral formalism, which does not single out time. A key result is a path integral representation of $Z(J)$, the generating function of $N$-body bulk wavefunctions. This is accomplished by introducing a time dependent chemical potential that changes abruptly at some Euclidean time. This procedure only works for particle nonconserving problems, hence the restriction to superconductors. We then drop some high derivative terms which do not matter in the infrared, and express $Z(J)$ as a Grassmann integral over a Lorentz invariant action. Rotating by 90 degrees to exchange time and a spatial direction we obtain the same topological superconductor but with a spatial edge induced by the jump in chemical potential. We find that the same $Z(J)$ has now morphed into the generating

This is an Open Access article distributed under the terms of the Creative Commons Attribution-Noncommercial License 3.0, which permits unrestricted use, distribution, and reproduction in any noncommercial medium, provided the original work is properly cited. 
function for the edge correlation functions. Three examples are given: the $p+i p$ superconductor in $D=2+1,{ }^{3}$ He B phase in $D=3+1$ and a p-wave superconductor (the Ising model) in $D=1+1$.

\section{$2 Z(J)$ : The generator of wave functions}

Recall that given a second-quantized $N$-body state $|\Phi\rangle$ with wavefunction $\phi\left(x_{1}, x_{2}, \ldots x_{N}\right)$ we extract $\phi$ using

$$
\phi\left(x_{1}, x_{2}, . . x_{N}\right)=\left\langle\emptyset\left|\Psi\left(x_{1}\right) \ldots \Psi\left(x_{N}\right)\right| \Phi\right\rangle .
$$

where $\langle\emptyset|$ is the Fock vacuum and $\Psi$ is the canonical electron destruction operator. For problems with variable number of particles, let us define the generating function

$$
Z(J)=\left\langle\emptyset\left|e^{\int d x J(x) \Psi(x)}\right| \Phi\right\rangle
$$

which yields $N$-body wavefunctions upon differentiating $N$ - times with respect to the Grassmann source $J(x)$.

The symbol $Z(J)$ is deliberate: one can write the generating function of $\mathrm{N}$-particle wavefunctions as a path integral or partition function. The Appendix gives a compact derivation of this fact and why the wavefunctions are Pfaffians. If all we want are the wavefunctions, this will do. But we shall now go on to write a different expression for $Z(J)$ that allows us to show the relation between bulk and edge.

We want to express $Z(J)$ as a path integral when $|\Phi\rangle$ is the ground state of a Hamiltonian $H$ without conserved particle number. Since Euclidean time evolution for long times projects to the ground state, we can obtain $|\Phi\rangle$ as

$$
|\Phi\rangle=U\left(0^{-},-\infty\right)|i\rangle
$$

where $|i\rangle$ is a generic initial state and $U\left(0^{-},-\infty\right)$ is the imaginary time propagator from $-\infty$ to $0^{-}$. Then we insert the operator $\exp \left[\int J(x) \Psi(x) d x\right]$ at time 0 . Finally, we obtain the Fock vacuum by evolving a generic state $\langle f|$ from time $+\infty$ to $0^{+}$using a hamiltonian $H^{\prime}$ with a huge negative $\mu$ that empties out fermions so that we may write $\langle\emptyset|=\langle f| U\left(\infty, 0^{+}\right)$. Thus

$$
Z(J)=\left\langle f\left|U\left(\infty, 0^{+}\right) e^{\int J(x) \Psi(x) d x} U\left(0^{-},-\infty\right)\right| i\right\rangle
$$

which has a path integral representation. 16]

Example 1: $p+i p$ : The mean-field hamiltonian is[17,

$$
H=\sum_{k}\left(c_{k}^{\dagger}, c_{-k}\right)\left(\begin{array}{cc}
\alpha k^{2}-\mu & \Delta \cdot\left(k_{1}-i k_{2}\right) \\
\Delta^{*} \cdot\left(k_{1}+i k_{2}\right) & -\left(\alpha k^{2}-\mu\right)
\end{array}\right)\left(\begin{array}{c}
c_{k} \\
c_{-k}^{\dagger}
\end{array}\right)
$$

where 1,2 are spatial indices and $x_{3}$ will be time. We retain the minimum $k$ dependence in the pairing function, and set the coefficient $\Delta=1$ so that the gap function is $\Delta\left(k_{1}, k_{2}\right)=k_{1}-i k_{2}$ Why then do we display the less relevant $\alpha k^{2}$ term in the kinetic energy? The answer is that without it, the physics is insensitive to the sign of $\mu$. But as Read and Green show, it is what allows us to associate the topologically trivial and nontrivial states with $\mu<0$ and $\mu>0$, with the latter containing fermions with momenta $\alpha k^{2} \leq \mu$. Once we bear in mind this connection between the sign of $\mu$ and the topology, we drop it in the subsequent computation of infrared wave functions.

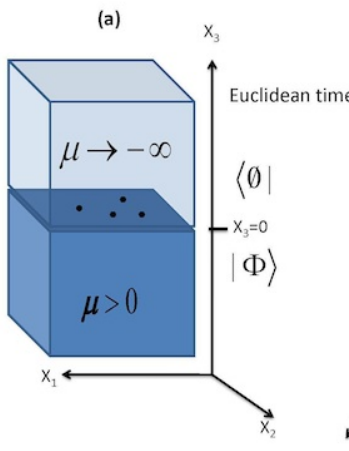

Bulk Wavefunction

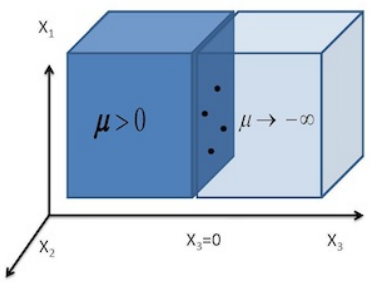

Edge Correlation
Fig. 2. Euclidean Rotation

Now the mean field Hamiltonian in real space

$$
H=\int d^{2} x\left[\Psi^{\dagger}(-\mu) \Psi+\frac{1}{2}\left(\Psi^{\dagger}\left(-i \partial_{1}-\partial_{2}\right) \Psi^{\dagger}+h . c\right)\right] \text {. }
$$

leads to corresponding Grassmann action for $U(0,-\infty)$ :

$$
\begin{aligned}
& S=\int_{-\infty}^{\infty} d^{2} x \int_{-\infty}^{0} d x_{3}[\bar{\psi} \mathcal{D} \psi+\bar{\psi} i \partial \bar{\psi}+\psi i \bar{\partial} \psi] \\
& \mathcal{D}=\left(-\partial_{3}+\mu\right) \quad \partial=\frac{\partial}{\partial z} \quad \bar{\partial}=\frac{\partial}{\partial \bar{z}}
\end{aligned}
$$

For the $0^{+}<x_{3}<\infty$, we choose $\mu=\mu_{+}$, a very large negative number, associated with the Fock vacuum and obtain, for all $x_{3}$, the action including the source $J$ :

$$
S(J)=\int_{-\infty}^{\infty} d^{3} x\left[\bar{\psi} \mathcal{D} \psi+\bar{\psi} i \partial \bar{\psi}+\psi i \bar{\partial} \psi+J \psi \delta\left(x_{3}\right)\right]
$$

where $\mathcal{D}$ now contains a time-dependent $\mu\left(x_{3}\right)$ that jumps at $x_{3}=0$ from $\mu_{-}>0$ to $\mu_{+} \rightarrow-\infty$.

The generating function of the BCS wavefunctions is

$$
Z(J)=\frac{\int[d \bar{\psi} d \psi] e^{S(J)}}{\int[d \bar{\psi} d \psi] e^{S(0)}}
$$

The story is depicted in the left half of Figure 2: the fermions travel unsuspectingly along in Euclidean time $x_{3}$ and slam like bugs onto the windshield at $x_{3}=0^{-}$when $\delta\left(x_{3}\right) J \psi$ kills them.

Since $\psi$ and $\bar{\psi}$ in Eq. 9 are independent Grassmann variables, we integrate out $\bar{\psi}$ to obtain the effective action for just $\psi$ to which alone $J$ couples:

$$
\begin{aligned}
S_{e f f}(\psi, J) & =\int d^{3} x\left(\psi i \bar{\partial} \psi+J \psi+\psi \frac{1}{4 i \partial} \mathcal{D}^{T} \mathcal{D} \psi\right) \\
& \equiv S_{0}(J)+S_{\text {ind }} .
\end{aligned}
$$

For the infrared limit we keep just the Jackiw-Rebbi zero mode [18] of the hermitian operator

$$
\mathcal{D}^{T} \mathcal{D}\left(x_{3}\right)=\left(\partial_{3}+\mu\left(x_{3}\right)\right)\left(-\partial_{3}+\mu\left(x_{3}\right)\right),
$$

that obeys $\mathcal{D} f_{0}=0$

$$
f_{0}\left(x_{3}\right)=f_{0}(0) e^{\int_{0}^{x_{3}} \mu\left(x^{\prime}\right) d x^{\prime}}
$$


in the mode expansion of the Grassmann field:

$$
\psi\left(x_{1}, x_{2}, x_{3}\right)=f_{0}\left(x_{3}\right) \psi\left(x_{1}, x_{2}\right) .
$$

This kills $S_{\text {ind }}$, and upon integrating $f_{0}^{2}$ over $x_{3}$,

$$
S_{e f f}(J)=\int d x_{1} d x_{2} \psi\left(i \bar{\partial}+J f_{0}(0)\right) \psi
$$

While this is indeed the action of a chiral majorana fermion living in the $1-2$ plane we are not done: we need to show that this fermion and this action also arise at the edge of the same $p+i p$ system. But so far we have no edge! It will be introduced shortly, but first a summary of results on the wavefunction.

\subsection{The Pfaffian Wavefunction:}

Integrating over $\psi$ in Eq. 15, and suppressing the constant $\left.f_{0}^{2}(0)\right)$ we find

$$
Z(J)=\exp \left[\int d^{2} \mathbf{r} J(\mathbf{r})\left[\frac{1}{4 i \bar{\partial}}\right]_{\mathbf{r r}^{\prime}} J\left(\mathbf{r}^{\prime}\right)\right]
$$

The two-particle wavefunction $\phi\left(\mathbf{r}_{1}-\mathbf{r}_{2}\right)$ can be written in terms of many related quantities:

$$
\phi=\frac{\partial^{2} Z(J)}{\partial J_{1} \partial J_{2}}=\left[\frac{1}{2 i \bar{\partial}}\right]_{\mathbf{r}_{1} \mathbf{r}_{2}}=\Delta_{\mathbf{r}_{1} \mathbf{r}_{2}}^{*-1}=\frac{1}{z_{1}-z_{2}}
$$

and the $N$-particle wavefunction is $\operatorname{Pf}\left(\frac{1}{z_{i}-z_{j}}\right)$. In the Appendix we relate $Z(J)$ and the conventional BCS wavefunction:

$$
|B C S\rangle=\exp \left(\frac{1}{2} \int \Psi^{\dagger}(x) g(x-y) \Psi^{\dagger}(y) d x d y\right)|\emptyset\rangle
$$

and see that $\phi=-g\left(\mathbf{r}_{1}-\mathbf{r}_{2}\right)$.

\section{The Edge}

To relate $Z(J)$ in Eqn. 9 to a problem with the edge we rewrite $S(J)$ in Lorentz invariant form:

$$
\begin{aligned}
S(J) & =\int d^{3} x\left[\bar{\Psi}(\not \partial-\mu) \Psi+J^{T} \Psi\right] \text { where } \\
\Psi & =\left(\begin{array}{c}
\psi \\
\bar{\psi}
\end{array}\right) \quad \bar{\Psi}=\Psi^{T} \varepsilon ; \quad \varepsilon=i \sigma_{2} \quad \not \partial=\gamma_{\mu} \partial_{\mu} \\
\gamma_{1} & =\sigma_{2} \quad \gamma_{2}=-\sigma_{1} \quad \gamma_{3}=\sigma_{3} \\
J^{T} & =J \delta\left(x_{3}\right)(10) .
\end{aligned}
$$

Look at the left half of Figure 1. We see our current description of the superconductor: translationally invariant in the $x_{1}-x_{2}$ plane, regarded as the space in which the $p_{1}+i p_{2}$ superconductor lives, and with a jump in $\mu$ at "time" $x_{3}=0$. In this description, the functional integral is saturated by one mode $f_{0}\left(x_{3}\right)$, glued to the interface, exactly like the electron gas at a heterojunction.

Extracting $H\left(x_{1}, x_{2}\right)$ from the Lorentz invariant action is like taking the row-to row transfer matrix. To derive the hamiltonian that governs the column-to -column dynamics, we rotate the three dimensional spacetime by $-\frac{\pi}{2}$ around the $x_{2}$ axis to obtain the view shown in the right half of Figure 2. The points carry the same labels as before but the spinor undergoes a rotation:

$$
\Psi=\left(\begin{array}{c}
\psi \\
\bar{\psi}
\end{array}\right)=e^{i \frac{\pi}{4} i \gamma_{3} \gamma_{1}}\left(\begin{array}{c}
\psi^{\prime} \\
\bar{\psi}^{\prime}
\end{array}\right)=e^{i \frac{\pi}{4} \sigma_{1}} \Psi^{\prime}
$$

Upon performing this transformation we end up with

$$
\begin{aligned}
S\left(\Psi^{\prime}, J\right) & =\int d^{3} x\left[\bar{\Psi}^{\prime}\left[\sigma_{3} \partial_{1}-\sigma_{1} \partial_{2}-\sigma_{2} \partial_{3}-\mu\right] \Psi^{\prime}\right. \\
& \left.+J \delta\left(x_{3}\right)\left(\frac{\psi^{\prime}+i \bar{\psi}^{\prime}}{\sqrt{2}}\right)\right]
\end{aligned}
$$

which describes exactly the same $p+i p$ superconductor but in the $2-3$ plane (with $1 \rightarrow 3,3 \rightarrow-1$ ) with an edge at $x_{3}=0$ with the $\mu>0$ side containing the nontrivial superconductor.

To see that the field $\frac{\psi^{\prime}+i \bar{\psi}^{\prime}}{\sqrt{2}}$ that $J$ couples to is precisely the Majorana field that arises at the edge, consider solving the equation for the zero mode which follows from Eq. 24 on dropping all $x_{1}, x_{2}$ dependence:

$$
\left(\sigma_{2} \partial_{3}+\mu\left(x_{3}\right)\right) \chi_{0}^{\prime}=0 \Rightarrow \chi_{0}^{\prime}\left(x_{3}\right)=\frac{1}{\sqrt{2}}\left(\begin{array}{c}
1 \\
-i
\end{array}\right) f_{0}\left(x_{3}\right)
$$

the normalizable spinor solution indeed corresponds to the operator $\frac{1}{\sqrt{2}}\left(\psi^{\prime}+i \psi^{\prime \dagger}\right)$.

We are done, for we have shown that $Z(J)$ is at once the generators of electronic wavefunction in the bulk and of correlation functions of the Majorana field at the edge.

For completeness, the edge Majorana field action follows from saturating the $x_{3}$ dependence of $\Psi^{\prime}$ as follows:

$$
\Psi^{\prime}\left(x_{1}, x_{2}, x_{3}\right)=\frac{1}{\sqrt{2}}\left(\begin{array}{c}
1 \\
-i
\end{array}\right) f_{0}\left(x_{3}\right) \psi^{\prime}\left(x_{1}, x_{2}\right)
$$

Plugging this into the action $S\left(\Psi^{\prime}, J\right)$ one finds, upon integrating the normalized function $f_{0}^{\prime}\left(x_{3}\right)$ over $x_{3}$

$$
S\left(\Psi^{\prime}, J\right) \rightarrow \int d x_{1} d x_{2}\left[\psi^{\prime} i \bar{\partial} \psi^{\prime}+J f_{0}(0) \psi^{\prime}\right]
$$

exactly as in Eqn.15, for the wavefunction.

\section{$4{ }^{3} \mathrm{He}-\mathrm{B}$ in $\mathrm{D}=\mathbf{3}+\mathbf{1}:$}

In a simplified model of superfluid ${ }^{3} \mathrm{He}-\mathrm{B}$, Cooper pairs have spin 1, whose projection lies perpendicular to the momenta $\pm \mathbf{k}[20,21]$. The winding of this axis around the Fermi surface in the weak pairing phase leads to its topological properties[21,22]. The mean-field Hamiltonian for this time-reversal invariant class DIII system is [20,21] is:

$$
\begin{aligned}
H= & \sum_{\mathbf{p} \sigma \sigma^{\prime}} \Psi_{\mathbf{p} \sigma}^{\dagger}\left(\frac{\mathbf{k}^{2}}{2 m}-\mu\right) \Psi_{\mathbf{k} \sigma} \\
& +\left\{\Delta_{\mathbf{k} \sigma \sigma^{\prime}} \psi_{\mathbf{k} \sigma} \psi_{-\mathbf{k} \sigma^{\prime}}+\text { h.c. }\right\} \\
\Delta_{\mathbf{k} \sigma \sigma^{\prime}}= & {[\varepsilon \mathbf{k} \cdot \boldsymbol{\sigma}]_{\sigma \sigma^{\prime}} }
\end{aligned}
$$


The $d=3$ problem is just the $d=2$ problem on steroids: $\Delta$ goes from being a complex number to a quaternion, and the spinless fermion is replaced by a two-component spinor. Hence the weak-pairing wavefunction is

$$
g_{\sigma_{i} \sigma_{j}}\left(\mathbf{r}_{i j}\right) \sim \frac{\left[\mathbf{r}_{i j} \cdot \boldsymbol{\sigma} \varepsilon\right]_{\sigma_{i} \sigma_{j}}}{r_{i j}^{3}}
$$

and the many-body wavefunction is $\operatorname{Pf}(g)$, as in $\operatorname{Ref}$. [9]:

$$
\Psi\left(\mathbf{r}_{1} \sigma_{1}, \mathbf{r}_{2} \sigma_{2}, \ldots, \mathbf{r}_{2 N} \sigma_{2 N}\right)=\operatorname{Pf}\left\{g_{\sigma_{i} \sigma_{j}}\left(\mathbf{r}_{i}-\mathbf{r}_{j}\right)\right\}
$$

The Lorentz invariant action for the wavefunction is

$$
\begin{aligned}
S & =\int d^{4} x \frac{1}{2} \bar{\Psi}[\not \partial-\mu] \Psi \quad \text { where } \\
\gamma_{0} & =\left(\begin{array}{cc}
I & 0 \\
0 & -I
\end{array}\right) \quad \gamma=\left(\begin{array}{cc}
0 & i \sigma \varepsilon \\
i \varepsilon \sigma & 0
\end{array}\right) \\
\bar{\Psi} & =\Psi^{T}\left(\begin{array}{cc}
0 & I \\
-I & 0
\end{array}\right)
\end{aligned}
$$

Now the 0 and 1 directions are exchanged by $R=$ $\exp \left[\frac{i \pi}{2} \frac{i \gamma_{0} \gamma_{1}}{2}\right]$, so that $J$ now couples to $\frac{\psi^{\prime}+i \sigma_{3} \psi^{\prime \prime}}{\sqrt{2}}$ which is readily verified, as before, to be the gapless edge mode of the rotated theory. The action for the edge theory obtained by saturating with the zero mode is

$$
S_{\text {edge }}=\int d^{3} x \frac{1}{2} \bar{\psi} \not \partial \psi \quad \not \partial=\sigma_{j} \partial_{j} \quad \bar{\psi}=\psi^{T}\left(-\sigma_{2}\right)
$$

\section{The Kitaev superconductor in $d=1$}

We could equally well go down a dimension, to a spinless p-wave superconductor in $d=1+1[15]$ where $\Delta=k_{x}$, which is also related to the quantum Ising model, via the Jordan-Wigner mapping. The edge theory is $0+1$ dimensional, corresponding to a Majorana zero mode, with $\mathcal{L}=$ $\frac{1}{2} \psi \partial_{x} \psi$. The pair wavefunction in the weak pairing phase is $g(x) \sim \operatorname{Sign}(x)$.

\section{Fractionalized Topological Superconductors:}

Our discussions thus far have been restricted to free fermion phases. In this section we indicate how analogs of fractional quantum Hall states, fractionalized topological superconductors, can be handled within our formalism. We construct a fractionalized superconducting phase in $\mathrm{D}=2+1$ that bears the same relation to the $p+i p$ superconductor as the Laughlin $m=3$ quantum Hall state bears to the integer Hall effect. Consider splitting the electron operator at each site into three fermions ('partons') $c_{r}=i f_{1 r} f_{2 r} f_{3 r}$ and $c_{r}^{\dagger}=i f_{1 r}^{\dagger} f_{2 r}^{\dagger} f_{3 r}^{\dagger}[29,30]$ with the following $p+i p$ mean field action for the partons:

$$
\begin{aligned}
S(J) & =\int d^{3} x\left[\mathcal{L}_{0}+i J f_{1} f_{2} f_{3}\right] \\
\mathcal{L}_{0} & =\frac{1}{2} \sum_{a=1}^{3}\left(\bar{f}_{a} f_{a}\right)\left(\begin{array}{l}
-\partial_{3}+\mu i \partial_{1}+\partial_{2} \\
i \partial_{1}-\partial_{2}-\partial_{3}-\mu
\end{array}\right)\left(\begin{array}{l}
f_{a} \\
\bar{f}_{a}
\end{array}\right)
\end{aligned}
$$

When the gauge theory is in a deconfined phase, the partons accurately describe the low energy dynamics. The $\mathrm{SO}(3)$ symmetry of the action, a remnant of the $\mathrm{SU}(3)$ gauge redundancy implied by $c_{r}=i f_{1 r} f_{2 r} f_{3 r}$ [29], is the gauge symmetry here. When the gapped bulk is integrated out, it generates an $\mathrm{SO}(3)_{1}$ (or equivalently $\left.\mathrm{SU}(2)_{2}\right)$ [31] ChernSimons term which renders the gauge field massive thereby liberating the partons with the action in Eq. 35. Note, the nontrivial part of this effective action is identifying the electron operator with a product of three $f$ fermions - which is manifested in the source $J$ coupling.

Emptying out the electrons requires removing the $f$ fermions, by taking their chemical potential to be large and negative, leading to the Fock vacuum. The electron correlators involve products of three parton correlators each in a $\mathrm{p}+\mathrm{ip}$ state, so the electronic wavefunction is:

$$
\Psi\left(z_{1}, z_{2}, \ldots, z_{2 N}\right)=\left\{\operatorname{Pf}\left[\frac{1}{z_{i}-z_{j}}\right]\right\}^{3}
$$

Note, this wavefunction is to be used in the infrared, when particles are well separated.

Equivalently we can start from the edge where the three Majorana modes are massless by gauge symmetry and have no relevant short range interactions in three spacetime dimensions. Long range gauge interactions do not exist due to the Chern-Simons term. Consequently the effective action that generates the bulk wavefunction must also be described by three noninteracting fermions.

\section{Summary:}

We have explained why the electronic wavefunctions in the bulk coincided with the massless Majorana correlation functions at the edge in certain problems. We first wrote $Z(J)=\left\langle\emptyset\left|e^{J \Psi}\right| B C S\right\rangle$ as a path integral in which the chemical potential abruptly jumped at in Euclidean time. Dropping the ' $k$ ', terms, but not the connection they provide between the sign of $\mu$ and topology, we obtained a Lorentz invariant action. Upon rotation by $\pi / 2$ the same action described a system that had an edge and $Z(J)$ had meanwhile morphed into the generating function for edge correlations.

While our trick of rotating the axes can be tried in any Euclidean path integral, Lorentz invariance is needed to ensure that the bulk for which the wavefunction is written is the same as the one with the edge after rotation. For Laughlin states realized by applying a magnetic field to fermions with a parabolic dispersion, we run into two kinds of problems: the action is far from Lorentz invariant and we cannot vary $\mu$ to drain the sea of particles since their number is conserved. We are working on deriving the appropriate bulk-boundary connection.

The bulk-boundary correspondence presented here is not limited to $D=2+1$ and is based on the approximate Lorentz invariance of the mean-field action. It is very different from that of topological Chern-Simons theories with vanishing hamiltonian and restricted to $D=2+1$.

Various topological superconductors are known corresponding to different Altland-Zirnbauer classes[9] - can our method be applied to any of them? In order to drain the Fermi sea in our derivation, the band structure should itself be trivial, and all the topology must be contained in the pairing (such as the phase winding around the Fermi 
surface in $p+i p)$. Such a construction is possible for topological phases in class $\mathrm{D}$ in $\mathrm{d}=1$ and $\mathrm{d}=2$ (like $\mathrm{p}+\mathrm{ip}$ ), in class $\mathrm{C}$ in $\mathrm{d}=2$ (like $\mathrm{d}+\mathrm{id}$ ) and class DIII in $\mathrm{d}=2,3(\mathrm{He}-3 \mathrm{~B}$ phase). However, it appears to be not possible for class CI in $\mathrm{d}=3,[27]$ which additionally rely on non-trivial topology of the weak pairing Fermi surface.

The entanglement spectrum of the bulk seems to determine the edge theory $[25,26]$, which we now relate back to the bulk wavefunction. Since the entanglement of a gapped phase appears from near the cut, the entire bulk wavefunction must be coded holographically in every $d-1$ dimensional sliver probed in the entanglement analysis.

Previously, the connection between edge states and bulk wavefunctions has played an important role identifying new FQH states[11,28]. Our work suggests a similar approach could be fruitful in identifying interacting topological phases in $\mathrm{D}=3+1$.

We thank the NSF for grants DMR- 0645691 (AV), and DMR- 0103639 (RS).

\section{Appendix: Majorana integrals and Pfaffian wavefunctions}

Suppose we are given a Majorana hamiltonian

$$
H=\frac{1}{2} \sum_{i j} \Psi_{i} h_{i j} \Psi_{j}
$$

wherein

$$
\left\{\Psi_{j}, \Psi_{j}\right\}=\delta_{i j},
$$

where $i, j$ subsume all labels, spatial and internal. If the labels are continuous, the Dirac $\delta$ should be used and derivatives $\partial$ viewed as antisymmetric matrices.

By definition the Grassmann integrals are

$$
\int \psi d \psi=1 \quad \int 1 \cdot d \psi=0
$$

The Euclidean path integral corresponding to $h$ is

$$
Z=\int[d \psi] e^{\frac{1}{2} \int d t \sum_{i j} \psi_{i}(t)\left(-\partial_{t} \delta_{i j}-h_{i j}\right) \psi_{j}(t)}
$$

Since coherent states do not exist for Majorana operators (which square to $\frac{1}{2}$ and not 0 ), one way to derive this result is to first form Dirac operators from pairs of Majorana operators, use fermion coherent states for the former to obtain a path integral and then undo the transformation back to Majorana fields.

The Gaussian integral that is repeatedly used is

$$
Z(J)=\int e^{\frac{1}{2} \chi A \chi+J \chi}[d \chi]=P f(A) e^{\frac{1}{2} J A^{-1} J}
$$

where $J$ and $\chi$ are $2 N$-component Grassmann vectors.

The two-point correlator is

$$
\left\langle\psi_{a} \psi_{b}\right\rangle=\left.\frac{\partial^{2} Z(J)}{\partial J_{a} \partial J_{b}}\right|_{J=0}=A_{b a}^{-1}=-A_{a b}^{-1}
$$

The minus sign in the last term can be avoided if the exponent is written as $e^{-\frac{1}{2} \chi A \chi}$.

Higher correlators are given by Pfaffians.

\subsection{Pfaffian wavefunctions}

Let us put these ideas to work in deriving the many-body wave functions from the second quantized BCS state.

Consider the generating function of wavefunctions for any number of particles from which the wavefunctions can be obtained by differentiating with respect to the Grassmann source $J(x)$

$$
\begin{aligned}
& Z(J)= \\
& \quad\left\langle\emptyset\left|e^{\int d x J(x) \Psi(x)}\right| B C S\right\rangle \\
& =\left\langle\emptyset\left|e^{\int d x J(x) \Psi(x)} \cdot I \cdot e^{\frac{1}{2} \int \Psi^{\dagger}(x) g(x-y) \Psi^{\dagger}(y) d x d y}\right| \emptyset\right\rangle \\
& \equiv \int[d \bar{\psi} d \psi] e^{-\bar{\psi} \psi}\left\langle\emptyset\left|e^{J(x) \Psi}\right| \psi\right\rangle\left\langle\bar{\psi}\left|e^{\frac{1}{2} \Psi^{\dagger} g \Psi^{\dagger}}\right| \emptyset\right\rangle
\end{aligned}
$$

where, in the last step we have resorted to a compact notation and inserted the following resolution of the identity in terms of Grassmann coherent states:

$$
I=\int|\psi\rangle\langle\bar{\psi}| e^{-\bar{\psi} \psi}[d \bar{\psi} d \psi]
$$

and where it is understood for example that

$$
|\psi\rangle=\prod_{x}|\psi(x)\rangle \quad[d \bar{\psi} d \psi]=\prod_{x}[d \bar{\psi}(x) d \psi(x)]
$$

It is important to remember that $\bar{\psi}$ and $\psi$ are independent and dummy variables. Using the defining property of coherent states

$$
\Psi|\psi\rangle=\psi|\psi\rangle \quad\langle\bar{\psi}| \Psi^{\dagger}=\langle\bar{\psi}| \bar{\psi}
$$

in Eq. 43 we find

$$
Z(J)=\int[d \bar{\psi} d \psi] e^{-\bar{\psi} \psi} e^{J \psi} e^{\frac{1}{2} \bar{\psi} g \bar{\psi}}
$$

where we have used the fact that

$$
\langle\emptyset \mid \psi\rangle\langle\bar{\psi} \mid \emptyset\rangle=1
$$

since at each site

$$
|\psi\rangle=|0\rangle-\psi|1\rangle \quad\langle\bar{\psi}|=\langle 0|-\langle 1| \bar{\psi}
$$

and $|\emptyset\rangle=|0\rangle \otimes|0\rangle \otimes \ldots . .|0\rangle$. Doing the integrals over $\psi$ and $\bar{\psi}$, we find

$$
Z(J)=e^{\frac{1}{2} J g J} .
$$

The pair wavefunction is

$$
\phi\left(x_{1}, x_{2}\right)=\left.\frac{\partial^{2} Z}{\partial J\left(x_{1}\right) \partial J\left(x_{2}\right)}\right|_{J=0}=-g\left(x_{1}-x_{2}\right)
$$

Higher correlations follow from Wick's theorem. For example

$$
\begin{aligned}
\phi\left(x_{1}, x_{2}, x_{3}, x_{4}\right) & =g\left(x_{1}-x_{2}\right) g\left(x_{3}-x_{4}\right) \\
& -g\left(x_{1}-x_{3}\right) g\left(x_{2}-x_{4}\right) \\
& +g\left(x_{1}-x_{4}\right) g\left(x_{2}-x_{3}\right) .
\end{aligned}
$$




\section{References}

1. R.Shankar and Ashvin Vishwanath, Phys. Rev. Lett., 107, 106803, (2011).

2. B. I. Halperin, Phys. Rev B 25, 2185, (1982), Helv. Phys. Acta 56, 75, (1983).

3. X.G. Wen, Phys. Rev. B 41, 12838, Intl. J. Mod. Phys. B 6 (10), 1711, (1990).

4. F.D.M.Haldane, Phys. Rev. Lett. 74, 2090, (1995).

5. C.L. Kane and E.J. Mele, Phys. Rev. Lett. 93, 197402, (2004), L. Fu, C.L. Kane and E.J. Mele, Phys. Rev. Lett. 98, 106803 (2007).; M. Z. Hasan and C. L. Kane, Rev. Mod. Phys. 82, 3045 (2010).

6. B. A. Bernevig, T. L. Hughes, and S.C. Zhang, Science 314, 1757 (2006), X. Qi and S. Zhang, Physics Today 63, 33, (2010); X.-L. Qi, T. L. Hughes, and S.C. Zhang, Phys. Rev. B 78, 195424 (2008), X.-L. Qi and S.-C. Zhang, Topological insulators and superconductors, e-print arXiv:1008.2026 (2010), to appear in Rev. Mod. Phys.

7. J. Moore and L. Balents, Phys. Rev. B 75, 121306, (2007); J. Moore, Nature 464, 194 (2010).

8. R. Roy, Phys. Rev. B 79, 195322, (2009).

9. A.P. Schnyder, S. Ryu, A. Furusaki, and A. W. W. Ludwig, Phys. Rev. B 78, 195125, (2008) and New J. Phys. 12065010 doi: 10.1088/13672630/12/6/065010, (2010).

10. A. Kitaev, Periodic table for topological insulators and superconductors (2009), arXiv:0901.2686.

11. G. Moore and N. Read, Nucl. Phys. B 360(2-3), 362 (1991).

12. M. Greiter, X.-G. Wen, and F. Wilczek, Phys. Rev. Lett. 66(24) , 3205, (1991) and Nucl. Phys. B 374(3), 567, (1992).

13. C. Nayak, S. H. Simon, A. Stern, M. Freedman and S. Das Sarma, Rev. Mod. Phys., 80 , 1159, (2008).

14. E. Witten, Comm. Math. Phys. 121, 351, (1989).

15. Alexei Kitaev, Unpaired Majorana fermions in quantum wires (2000), arXiv:cond-mat/0010440.

16. N. Read and D. Green, Phys. Rev. B 61(15), 10267, (2000).

17. G.E. Volovik, The Universe in a Helium Droplet, Clarendon, Oxford, (2003).

18. R. Jackiw, and C. Rebbi, Phys. Rev. D 13, 3398, (1976).

19. X. G. Wen, Phys. Rev. B60, 8827, (1999).

20. A. J. Leggett, Rev. Mod. Phys. 47, 331 (1975).

21. D. Vollhardt and P. Wolfle, The Superfluid Phases of Helium 3 (Taylor and Francis, USA, 1990).

22. R. Shankar, J. de Physique 38, 1405, (1977).

23. X.G. Wen, Phys. Rev. B65, 165113 (2002).

24. J. Maciejko, X.-L. Qi, A. Karch, and S.-C. Zhang, Phys. Rev. Lett., 105, 2468, (2010), B. Swingle, M. Barkeshli, J. McGreevy, T. Senthil, Correlated Topological Insulators and the Fractional Magnetoelectric Effect, Fractional topological insulators in three dimensions, arXiv:1005.1076. M. Levin and A. Stern, Phys. Rev. Lett. 103, 196803 (2009).

25. H. Li and F. D. M. Haldane, Phys. Rev. Lett. 101, 246806, (2008).

26. L. Fidkowski, Phys. Rev. Lett. 104, 130502, (2010). A. Turner, Y. Zhang, A. Vishwanath, Phys. Rev. B 82, 241102R, (2010). X-L. Qi, H. Katsura, and A. W. W. Ludwig, General Relationship Between the Entangle- ment Spectrum and the Edge State Spectrum of Topological Quantum States, arXiv:1103.5437v1.

27. A. P. Schnyder, S. Ryu, A. W. W. Ludwig, Phys. Rev. Lett. 102, 196804 (2009).

28. N. Read and E. Rezayi, Phys.Rev. B59, 8084 (1999).

29. X.G. Wen, Phys. Rev. B65, 165113 (2002).

30. J. Maciejko, X.-L. Qi, A. Karch, and S.-C. Zhang, Phys. Rev. Lett., 105, 2468, (2010), B. Swingle, M. Barkeshli, J. McGreevy, T. Senthil, Correlated Topological Insulators and the Fractional Magnetoelectric Effect, Fractional topological insulators in three dimensions, arXiv:1005.1076. M. Levin and A. Stern, Phys. Rev. Lett. 103, 196803 (2009).

31. E. Fradkin, C. Nayak, A. Tsvelik, F. Wilczek Nucl.Phys. B516, 704, (1998). H. Yao, D. H. Lee, Fermionic magnons, non-Abelian spinons, and spin quantum Hall effect from an exactly solvable Kitaev Hamiltonian with SU(2) symmetry, arXiv:1010.3724. 